\title{
Adubação potássica na qualidade de frutos de pessegueiro
}

\author{
Potassium fertilization in peach fruit quality
}

\author{
Caroline Farias Barreto ${ }^{1 *}$, Letícia Vanni Ferreira ${ }^{2}$, Renan Navroski ${ }^{3}$, Jorge Atílio Benati ${ }^{3}$, Rufino \\ Fernando Flores Cantillano', Marcia Vizzotto ${ }^{1}$, Gilberto Nava ${ }^{1}$, Luis Eduardo Correa Antunes ${ }^{1}$ \\ ${ }^{1}$ Embrapa Clima Temperado Pelotas, RS, Brasil. *Autor para correspondência: carol_fariasb@hotmail.com \\ ${ }^{2}$ Instituto Federal de Educação, Ciência e Tecnologia do Rio Grande do Sul, Ibirubá, RS, Brasil. \\ 3Universidade Federal de Pelotas, Pelotas, RS, Brasil.
}

Submissão: 04/04/2020 / Aceite: 02/09/2020

\begin{abstract}
RESUMO
Para pessegueiros produzidos na região sul do Brasil ainda são escassas as informações sobre a influência da adubação potássica sobre a qualidade dos frutos. Deste modo, o presente trabalho teve como objetivo avaliar os atributos físico-químicos e compostos bioativos dos frutos de pessegueiros submetidos a diferentes doses de potássio aplicadas no solo por três safras consecutivas. O experimento foi realizado estudando a cultivar Sensação em um pomar comercial no município de Morro Redondo, RS - Brasil, durante as safras de 2016, 2017 e 2018. Os pessegueiros da cultivar Sensação foram adubados com potássio (K) nas doses de 0,40, 80, 120 e $160 \mathrm{~kg} \mathrm{ha}^{-1}$ de $\mathrm{K}_{2} \mathrm{O}$ na forma de cloreto de potássio, aplicadas na superfície do solo. Após a colheita foram determinados os teores de sólidos solúveis (SS), acidez titulável (AT), relação SS/AT, pH, coloração da polpa, firmeza da epiderme e da polpa, teor de carotenoides totais, compostos fenólicos totais e atividade antioxidante. A firmeza da epiderme, a relação SS/AT e a concentração de carotenoides dos pêssegos responderam à adubação potássica, mas apenas em alguns ciclos de produção. Os parâmetros de coloração, firmeza da polpa, sólidos solúveis, pH do suco, fenóis totais e atividade antioxidante dos pêssegos não alteraram em decorrência da aplicação de potássio ao solo.
\end{abstract}

PALAVRAS-CHAVE: físico-químicas, fitoquímicos, Prunus persica, nutrição mineral.

\begin{abstract}
For peach trees produced in southern Brazil, information about the influence of potassium fertilization on fruit quality remains scarce. This study set out to determine the physicochemical properties and bioactive compounds of peaches treated with different doses of potassium applied to the soil for three consecutive crop years. The experiment examined the Sensação cultivar in a commercial orchard in the municipality of Morro Redondo, Rio Grande do Sul, Brazil, during the 2016, 2017, and 2018 crop years. Peach trees of the Sensação cultivar were fertilized with potassium (K); doses of $0,40,80,120$, and $160 \mathrm{~kg} \mathrm{ha}^{-1}$ of $\mathrm{K}_{2} \mathrm{O}$ in the form of potassium chloride were applied to the soil surface. After harvest, an assessment was made of the soluble solids (SS) content, titratable acid (TA) content, SS/TA ratio, $\mathrm{pH}$ levels, flesh color, skin and flesh firmness, total carotenoid content, total phenolic content, and antioxidant activity. The peaches' skin firmness, SS/TA ratio, and concentration of carotenoids responded to potassium fertilization, but only in some production cycles. Their color, flesh firmness, soluble solids, juice $\mathrm{pH}$ levels, total phenols, and antioxidant activity did not change with the application of potassium in the soil.
\end{abstract}

KEYWORDS: physicochemical, phytochemicals, Prunus persica, mineral nutrition.

\section{INTRODUÇÃO}

Na comercialização de pêssegos é primordial a produção de frutos com alto padrão de qualidade, pois atributos como tamanho, cor, sabor e ausência de defeitos atraem o consumidor e são fatores decisivos no momento da compra (TREVISAN et al. 2010). Essas características de qualidade dos frutos estão diretamente ligadas à genética da cultivar, às condições edafoclimáticas, ao uso de técnicas culturais como irrigação, podas, tratamentos fitossanitários, raleio (GONÇALVES et al. 2014, MAYER et al. 2019), bem como a adubação dos pomares (FERREIRA et al. 2018).

O manejo adequado da adubação em pomares de pessegueiros tem impacto direto sobre a 
produtividade e qualidade dos frutos. Para frutíferas de caroço, os principais elementos minerais que as plantas necessitam são o nitrogênio $(\mathrm{N})$ e o potássio $(\mathrm{K})$. O K desempenha funções específicas e essenciais nas plantas como a expansão celular, fotossíntese, síntese de proteínas, transporte e acúmulo de carboidratos (BRUNETTO et al. 2015, TAIZ et al. 2017), sendo o macronutriente mais exportado por meio dos frutos (ROMBOLÀ et al. 2012).

Em pessegueiro, a adubação potássica pode influenciar nos parâmetros de qualidade dos frutos como a coloração da epiderme e firmeza de polpa (TREVISAN et al. 2006), pois este nutriente está relacionado com reações de síntese e de ativação enzimas que contribuem diretamente para a maturação, síntese de açúcar e a manutenção do turgor celular (NAVA et al. 2008, LESTER et al. 2010, BRUNETTO et al. 2015).

No cultivo de frutíferas deve-se realizar o acompanhamento da fertilidade dos pomares, utilizando-se a análise foliar e de solo, aliada à diagnose visual. No entanto, muitas vezes, a adubação realizada pelos produtores de pêssegos ocorre de forma empírica ou por especulação da quantidade requerida pelas plantas. Deste modo, o produtor pode estar realizando a adubação dos pomares de forma inadequada, aplicando adubo onde os teores dos nutrientes no solo estão altos ou não realizando a aplicação onde existe a necessidade.

No Brasil, as informações sobre a influência da adubação potássica sobre a qualidade dos pêssegos destinados ao consumo in natura e para a indústria ainda são incipientes. Estudos da qualidade de pêssegos na pós-colheita em função da adubação, juntamente com as respostas fitotécnicas, podem orientar o manejo da fertilidade do solo e da nutrição mineral das plantas. Neste contexto, o presente trabalho teve como objetivo avaliar os atributos físico-químicos e compostos bioativos dos frutos de pessegueiros submetidos a diferentes doses de potássio aplicadas no solo por três safras consecutivas.

\section{MATERIAL E MÉTODOS}

O experimento foi conduzido nos anos de 2016, 2017 e 2018 em pomar comercial localizado no município de Morro Redondo, no estado do Rio Grande do Sul (latitude 31³1'49,3"S e longitude $52^{\circ} 35^{\prime} 39,8^{\prime \prime}$ ). A classificação do clima da região, conforme W. Köppen é do tipo "cfa" - clima sub tropical úmido, ou seja, temperado úmido com verões quentes (ALVARES et al. 2013).

O pomar de pessegueiro utilizado foi da cultivar Sensação, enxertada sobre o porta-enxerto Capdeboscq. O pomar foi implantado no ano 2009, com sistema de condução das plantas em vaso, espaçamento entre linhas de $5 \mathrm{~m}$ e entre plantas de $2 \mathrm{~m}$, totalizando-se 1.000 plantas $\mathrm{ha}^{-1}$. As análises físico-químicas do solo, realizadas antes da instalação do experimento, na camada de $0-20 \mathrm{~cm}$, apresentaram os seguintes resultados: $\mathrm{pH}$ em água de 5,8; $3,2 \mathrm{cmol}_{\mathrm{c}} \mathrm{dm}^{-3} \mathrm{H}+\mathrm{Al}$; $\mathrm{CTC}$ a pH $7,0=8,1$ $\mathrm{cmol}_{\mathrm{c}} \mathrm{dm}^{-3} ; 11,3 \mathrm{mg} \mathrm{dm}^{-3}$ de P (teor baixo); $101 \mathrm{mg} \mathrm{dm}^{-3}$ de $\mathrm{K}$ (teor alto); $3,7 \mathrm{cmol}_{\mathrm{c}} \mathrm{dm}^{-3}$ de Ca (teor alto); 0,9 $\mathrm{cmol}_{\mathrm{C}} \mathrm{dm}^{-3}$ de $\mathrm{Mg}$ (teor médio); $26 \%$ de argila e 2,2\% de matéria orgânica.

O delineamento experimental foi em blocos ao acaso, com quatro repetições. As unidades experimentais foram compostas por quatro plantas, porém somente as duas plantas centrais foram avaliadas. As doses de $\mathrm{K}$ utilizadas foram 0, 40, 80, 120 e $160 \mathrm{~kg} \mathrm{ha}^{-1}$ de $\mathrm{K}_{2} \mathrm{O}$ na forma de cloreto de potássio $\left(60 \%\right.$ de $\left.\mathrm{K}_{2} \mathrm{O}\right)$, aplicadas anualmente próximas à plena floração dos pessegueiros (metade do mês de julho), na faixa de projeção da copa das plantas, sobre a superfície do solo e sem incorporação. Todas as parcelas receberam doses iguais de nitrogênio $(N)$ e fósforo $(P)$, conforme quantidades e épocas de aplicação recomendadas pela CQFS-RS/SC (2016).

Após a colheita foram utilizados 20 frutos por parcela para a realização das avaliações das variáveis: coloração da polpa realizada na região equatorial dos frutos com o auxílio de colorímetro da marca Minolta, modelo CR-400, que fornece os parâmetros $L^{*} a^{*} b^{*}$, onde $L^{*}$ expressa a luminosidade $\left(L^{*}=0\right.$ é preto e $L^{*}=$ 100 é branco) e a tonalidade (ângulo Hue); firmeza de epiderme e da polpa, mensuradas utilizando o texturômetro modelo TA-XT Plus, marca Stable Micro Systens com ponteira P-2 de $2 \mathrm{~mm}$, força de $5 \mathrm{~g}$ e velocidade de $5 \mathrm{~m} \mathrm{~ms}^{-1}$, com os resultados expressos em Newtons (N); Sólidos solúveis (SS), medidos com refratômetro digital manual da marca ATAGO, modelo PAL-1, com resultados expressos em ${ }^{\circ}$ Brix; acidez titulável (AT), determinada com $10 \mathrm{ml}$ de polpa de fruta e adicionadas a $90 \mathrm{ml}$ de água destilada, onde posteriormente realizou-se a titulação da amostra com auxílio de uma bureta digital Brand $^{\circledR}$ contendo solução de hidróxido de sódio $(\mathrm{NaOH})$ a $0,1 \mathrm{~N}$ até atingir o ponto de viragem, que é o pH 8,1, com resultados expressos em gramas de ácido cítrico $100 \mathrm{~g}^{-1}$ de polpa; Relação sólidos solúveis/acidez titulável (SS/AT) foi determinada pelo quociente entre ambas variáveis; potencial hidrogeniônico $(\mathrm{pH})$, determinado através de método eletrométrico, com o auxílio de um potenciômetro ( $\mathrm{pHmêtro)} \mathrm{da} \mathrm{marca} \mathrm{Quimis,} \mathrm{modelo} \mathrm{Q400A.}$

Para determinação dos compostos bioativos da polpa dos pêssegos foram realizadas as seguintes 
análises: compostos fenólicos totais determinados por meio da reação com Folin-Ciocalteau, conforme o método adaptado de SWAIN \& HILLIS (1959), sendo o resultado expresso em mg de ácido clorogênico em $100 \mathrm{~g}$ de amostra; atividade antioxidante determinada, através do radical estável DPPH de acordo com o método de BRAND-WILLIANS et al. (1995) e resultados expressos $\mu \mathrm{g}$ equivalente trolox $100 \mathrm{~g}^{-1}$ de amostra; carotenoides determinados pelo método de TALCOTT \& HOWARD (1999) e resultados foram calculados a partir de uma curva padrão construída com $\beta$-caroteno e expressos em mg de $\beta$-caroteno $100 \mathrm{~g}^{-1}$. Os resultados foram submetidos à análise de variância e quando os efeitos foram significativos, foram ajustadas as equações de regressão, tendo-se testado os modelos linear e quadrático pelo teste $F(p<0,05)$. A análise estatística foi realizada com o auxílio do programa Sisvar software, versão 5.6 (FERREIRA 2014).

\section{RESULTADOS E DISCUSSÃO}

A adubação potássica nos pessegueiros não alteram a coloração e a luminosidade da polpa dos frutos, nos três anos avaliados (Tabela 1). Desta forma, sugere-se que os aspectos envolvidos na coloração dos pêssegos podem estar relacionados a outros fatores, como o genótipo (MAYER et al. 2008), manejo da poda verde (GONÇALVES et al. 2014) e incidência da luz sobre os frutos (ALCOBENDAS et al. 2013).

Tabela 1. Coloração, luminosidade e firmeza da polpa de frutos de pessegueiros submetidos a adubação com diferentes doses de potássio no município de Morro Redondo, RS, nos anos de 2016, 2017 e 2018.

Table 1. Flesh color, lightness, and firmness of peach fruit fertilized with different doses of potassium in the municipality of Morro Redondo, RS, in 2016, 2017, and 2018.

\begin{tabular}{|c|c|c|c|}
\hline $\begin{array}{c}\text { Doses de } \mathrm{K}_{2} \mathrm{O} \\
\left(\mathrm{kg} \mathrm{ha}^{-1}\right)\end{array}$ & $\begin{array}{c}\text { Coloração } \\
\text { da polpa ('ue) }\end{array}$ & $\begin{array}{l}\text { Luminosidade } \\
\text { da polpa }\left(L^{*}\right)\end{array}$ & $\begin{array}{c}\text { Firmeza da } \\
\text { polpa (N) }\end{array}$ \\
\hline \multicolumn{4}{|c|}{2016} \\
\hline 0 & 82,36 & 74,73 & 1,21 \\
\hline 40 & 82,32 & 73,47 & 1,21 \\
\hline 80 & 82,84 & 73,42 & 1,25 \\
\hline 120 & 82,67 & 73,11 & 1,14 \\
\hline 160 & 82,83 & 72,09 & 1,18 \\
\hline CV (\%) & 0,36 & 1,27 & 11,39 \\
\hline Linear & ns & ns & ns \\
\hline Quadrática & ns & ns & ns \\
\hline \multicolumn{4}{|c|}{2017} \\
\hline 0 & 82,83 & 72,65 & 2,09 \\
\hline 40 & 83,07 & 73,57 & 2,04 \\
\hline 80 & 82,97 & 73,14 & 2,05 \\
\hline 120 & 83,16 & 72,62 & 2,08 \\
\hline 160 & 83,04 & 73,20 & 2,06 \\
\hline CV (\%) & 0,38 & 1,04 & 9,91 \\
\hline Linear & ns & ns & ns \\
\hline Quadrática & ns & ns & ns \\
\hline \multicolumn{4}{|c|}{2018} \\
\hline 0 & 79,71 & 80,64 & 2,20 \\
\hline 40 & 80,21 & 81,66 & 2,29 \\
\hline 80 & 79,71 & 81,06 & 2,03 \\
\hline 120 & 79,88 & 79,83 & 2,53 \\
\hline 160 & 80,09 & 80,65 & 2,31 \\
\hline CV (\%) & 0,31 & 1,16 & 12,04 \\
\hline Linear & ns & ns & ns \\
\hline Quadrática & ns & ns & ns \\
\hline
\end{tabular}


Os valores de firmeza não foram alterados com as doses de $\mathrm{K}$ aplicadas no solo, independente do ano de avaliação (Tabela 1). Esses resultados estão de acordo com CUQUEL et al. (2011) e GAZOLLANETO et al. (2007), que não verificaram efeito da adubação potássica na firmeza de polpa de ameixas e pêssegos.

A adubação potássica influenciou na firmeza da epiderme dos frutos. Verificou-se comportamento quadrático nos dois primeiros anos para esta variável, onde as maiores firmezas foram observadas com as doses de 64,5 e $107,1 \mathrm{~kg} \mathrm{ha}^{-1}$ de $\mathrm{K}_{2} \mathrm{O}$ no solo para os anos de 2016 e 2017, respectivamente (Figura 1). No terceiro ano do estudo (2018), constatou-se aumento linear da firmeza da epiderme com o incremento de $\mathrm{K}$ no solo (Tabela 1). A firmeza da epiderme é um atributo importante para a manutenção da integridade dos frutos durante $o$ transporte e comercialização, favorecendo a resistência no manuseio e reduzindo a ruptura física dos mesmos (GANESHAMURTHY et al. 2011).

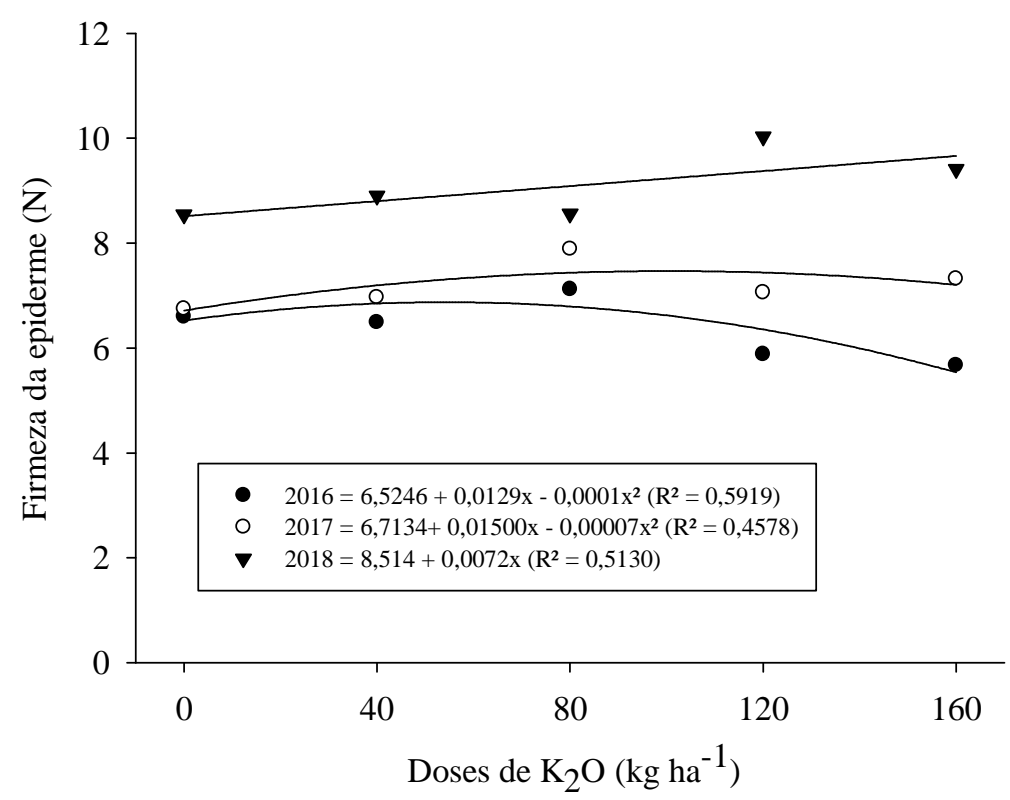

Figura 1. Firmeza da epiderme de frutos de pessegueiros submetidos à adubação com diferentes doses de potássio no município de Morro Redondo, RS, nos anos de 2016, 2017 e 2018.

Figure 1. Skin firmness of peach fruits fertilized with different doses of potassium in the municipality of Morro Redondo, RS, in 2016, 2017, and 2018.

Os sólidos solúveis dos frutos não foram influenciados pela adubação potássica nos pessegueiros, nos três anos do estudo (Tabela 2). Resultados similares foram verificados em pêssegos e ameixas por CHATZITHEODOROU et al. (2004) e CUQUEL et al. (2011). No entanto, na literatura é reportado que a adubação com $\mathrm{K}$ aumenta a concentração dos sólidos solúveis nos frutos (SOLHJOO et al. 2017, JAWANDHA et al. 2017), uma vez que este nutriente auxilia no transporte de carboidratos. Entretanto, é provável que o nível inicial de $\mathrm{K}$ no solo deste estudo (teor alto), tenha sido suficiente para a adequada nutrição das plantas.

Em relação à acidez titulável dos frutos, verificou-se comportamento linear decrescente com o incremento de $\mathrm{K}$ no solo somente no ano de 2016 (Tabela 2), corroborando com os resultados de DELGADO et al. (2004), os quais observaram que altas doses de $\mathrm{K}\left(120 \mathrm{~g} \mathrm{~K}_{2} \mathrm{O}\right)$ aplicadas no solo reduzem a acidez titulável de uvas. As variações do teor de ácidos nos frutos podem estar associadas à antecipação do período de amadurecimento dos frutos, favorecida pelo aumento dos teores de $\mathrm{K}$ disponíveis, de maneira que o amadurecimento pode ter elevado a degradação dos ácidos orgânicos, reduzindo assim a acidez da polpa dos frutos.

A relação SS/AT apresentou comportamento linear crescente em função do aumento das doses de $\mathrm{K}$ no solo no ano de 2016 (Tabela 2). Esta variável é calculada pela razão entre sólidos solúveis e acidez titulável, portanto no ano de 2016, como não houve efeito da adubação nos teores de sólidos solúveis, e a acidez titulável sofreu reduções com a adubação potássica, observou-se aumento nessa relação com o aumento das doses de $\mathrm{K}_{2} \mathrm{O}$ no solo. No entanto, no segundo ano do estudo (2017), a relação SS/AT obteve comportamento quadrático, onde os maiores valores desta variável foram observados com a dose de aproximadamente $56 \mathrm{~kg} \mathrm{ha}^{-1}$ de $\mathrm{K}_{2} \mathrm{O}$ no solo (Tabela 2). 
Tabela 2. Teores de sólidos solúveis (ํㅏix), acidez titulável (\% ácido cítrico), relação SS/AT e pH de frutos de pessegueiros submetidos à adubação com distintas doses de potássio no município de Morro Redondo, RS, nos anos de 2016, 2017 e 2018.

Table 2. Soluble solids content ( ${ }^{o}$ brix), titratable acid content (\% citric acid), SS/TA ratio, and pH levels of peach fruits fertilized with different potassium doses in the municipality of Morro Redondo, $R S$, in 2016, 2017, and 2018.

\begin{tabular}{|c|c|c|c|c|}
\hline Doses de $\mathrm{K}_{2} \mathrm{O}\left(\mathrm{kg} \mathrm{ha}^{-1}\right)$ & Sólidos solúveis & Acidez titulável & SS/AT & $\mathrm{pH}$ \\
\hline \multicolumn{5}{|c|}{2016} \\
\hline 0 & 8,82 & 1,14 & 7,70 & 3,58 \\
\hline 40 & 8,62 & 1,12 & 7,70 & 3,57 \\
\hline 80 & 8,50 & 1,06 & 8,01 & 3,62 \\
\hline 120 & 8,87 & 0,93 & 9,53 & 3,67 \\
\hline 160 & 8,50 & 1,00 & 8,54 & 3,67 \\
\hline CV (\%) & 3,82 & 7,78 & 6,2 & 1,68 \\
\hline Linear & ns & ${ }^{*}(1)$ & ${ }^{* *}(2)$ & ns \\
\hline Quadrática & ns & ns & ns & ns \\
\hline \multicolumn{5}{|c|}{2017} \\
\hline 0 & 9,80 & 1,69 & 5,78 & 3,53 \\
\hline 40 & 10,17 & 1,37 & 7,42 & 3,49 \\
\hline 80 & 9,97 & 1,71 & 5,79 & 3,54 \\
\hline 120 & 10,15 & 1,43 & 7,07 & 3,50 \\
\hline 160 & 10,02 & 1,81 & 5,55 & 3,54 \\
\hline CV (\%) & 6,64 & 4,43 & 7,79 & 0,76 \\
\hline Linear & ns & ns & ns & ns \\
\hline Quadrática & ns & ns & $* *(3)$ & ns \\
\hline \multicolumn{5}{|c|}{2018} \\
\hline 0 & 11,60 & 0,99 & 11,65 & 3,44 \\
\hline 40 & 11,52 & 1,12 & 10,28 & 3,41 \\
\hline 80 & 10,87 & 0,99 & 10,92 & 3,44 \\
\hline 120 & 12,15 & 1,13 & 10,72 & 3,42 \\
\hline 160 & 10,87 & 1,04 & 10,43 & 3,45 \\
\hline CV (\%) & 2,73 & 5,21 & 3,84 & 1,39 \\
\hline Linear & ns & ns & ns & ns \\
\hline Quadrática & ns & ns & ns & ns \\
\hline
\end{tabular}

ns não significativo para análise de regressão; significativo a $5 \%$ de probabilidade; significativo a $1 \%$ de probabilidade; ${ }^{(1)} y=1,144-0,0012 x\left(R^{2}=0,7363\right) ;{ }^{(2)} 7,594+0,088 x\left(R^{2}=0,5188\right) ;{ }^{(3)} y=5,9969+0,0223 x-0,0002 x^{2}\left(R^{2}=0,305\right)$.

$\mathrm{O}$ pH do suco dos pêssegos não foi influenciado pela adubação potássica dos pessegueiros nos três anos avaliados (Tabela 2). De acordo com ALCOBENDAS et al. (2013), este atributo nos pêssegos é mais dependente da orientação do fruto na planta, da exposição à luz solar e geralmente é reduzido quando ocorre aumento no diâmetro dos frutos, enquanto FERREIRA et al. (2016) concluíram que o pH do suco depende da cultivar de pessegueiro que está sendo avaliada.

Os teores de compostos fenólicos totais e a atividade antioxidante dos pêssegos não foram alterados pela adubação potássica nos pessegueiros nos três anos avaliados (Tabela 3). Desta forma, sugere-se que os teores desses compostos bioativos nos pêssegos podem variar de acordo com outros fatores genéticos da planta, como cultivar copa (SANTOS et al. 2013), porta-enxerto (BARRETO et al. 2017) e pela adubação nitrogenada dos pomares (FERREIRA et al. 2016).

Os teores de carotenoides dos pêssegos não foram alterados com as distintas doses de $\mathrm{K}$ aplicadas no solo, nos dois primeiros anos avaliados (2016 e 2017) (Tabela 3). No terceiro ano, os teores de carotenoides tiveram um comportamento quadrático os quais apresentaram os maiores valores com a dose 
de aproximadamente $48 \mathrm{~kg} \mathrm{ha}^{-1}$ de $\mathrm{K}_{2} \mathrm{O}$ aplicadas ao solo (Tabela 3). $\mathrm{O} \mathrm{K}$ pode desempenhar um papel importante no processo de biossíntese de carotenoides ativando várias enzimas que regulam o metabolismo dos carboidratos (FANASCA et al. 2006).

Tabela 3. Teores de compostos fenólicos totais, atividade antioxidante e carotenoides de frutos de pessegueiros submetidos à adubação com distintas doses de potássio no município de Morro Redondo, RS, nos anos de 2016, 2017 e 2018.

Table 3. Total phenolic content, antioxidant activity, and carotenoid content of peach fruit fertilized with different doses of potassium in the municipality of Morro Redondo, RS, in 2016, 2017, and 2018.

\begin{tabular}{|c|c|c|c|}
\hline Doses de $\mathrm{K}_{2} \mathrm{O}\left(\mathrm{kg} \mathrm{ha}^{-1}\right)$ & $\begin{array}{c}\text { Compostos } \\
\text { fenólicos totais }\end{array}$ & $\begin{array}{c}\text { Atividade } \\
\text { antioxidante }^{2}\end{array}$ & Carotenoides ${ }^{3}$ \\
\hline \multicolumn{4}{|c|}{2016} \\
\hline 0 & 177,69 & 2434,00 & 5,89 \\
\hline 40 & 210,57 & 2994,79 & 5,47 \\
\hline 80 & 162,81 & 2338,94 & 5,42 \\
\hline 120 & 165,60 & 2339,23 & 5,89 \\
\hline 160 & 197,83 & 2675,97 & 5,66 \\
\hline CV (\%) & 9,90 & 9,01 & 15,06 \\
\hline Linear & ns & ns & ns \\
\hline Quadrática & ns & ns & ns \\
\hline \multicolumn{4}{|c|}{2017} \\
\hline 0 & 93,87 & 971,70 & 4,15 \\
\hline 40 & 128,57 & 1080,62 & 4,31 \\
\hline 80 & 128,14 & 942,89 & 4,45 \\
\hline 120 & 97,62 & 706,78 & 4,89 \\
\hline 160 & 90,67 & 923,01 & 4,09 \\
\hline CV (\%) & 26,63 & 21,71 & 9,58 \\
\hline Linear & ns & ns & ns \\
\hline Quadrática & ns & ns & ns \\
\hline \multicolumn{4}{|c|}{2018} \\
\hline 0 & 264,06 & 1059,79 & 3,82 \\
\hline 40 & 250,06 & 965,41 & 4,94 \\
\hline 80 & 244,28 & 934,61 & 4,01 \\
\hline 120 & 276,39 & 1256,61 & 3,56 \\
\hline 160 & 266,39 & 1128,67 & 3,37 \\
\hline CV (\%) & 8,22 & 12,29 & 13,09 \\
\hline Linear & ns & ns & ns \\
\hline Quadrática & ns & ns & ${ }^{* *}(1)$ \\
\hline
\end{tabular}

De maneira geral, apenas algumas das variáveis qualitativas avaliadas nos pêssegos foram influenciadas pela adubação potássica nas três safras. Ressalta-se também neste trabalho o fato do teor inicial de $\mathrm{K}$ no solo ser alto, e possivelmente ter quantidade suficiente desse nutriente para suprir a demanda das plantas em relação aos atributos de qualidade dos pêssegos. Assim, evidencia-se que em solos que já possuem alto teor de $\mathrm{K}$, não existe a necessidade de realizar aplicação de fertilizantes potássicos almejando promover a qualidade de pêssegos.

As formas de aplicação do $\mathrm{K}$ também podem interferir nos atributos de qualidade dos pêssegos. Neste estudo, a aplicação de $\mathrm{K}$ ocorreu anualmente, via solo e sem incorporação. Em outros estudos envolvendo plantas frutíferas, a qualidade dos frutos foi alterada quando a aplicação de $\mathrm{K}$ ocorreu via foliar, enquanto a aplicação via solo de K resultou em pouco ou nenhum efeito (JIFON \& LESTER 2009, CUQUEL et al. 2011). 


\section{CONCLUSÃO}

A adubação potássica influencia a firmeza da epiderme dos pêssegos.

A coloração, firmeza da polpa, sólidos solúveis, pH do suco, compostos fenólicos totais e atividade antioxidante dos pêssegos não são alterados pela adubação potássica em solos com teor alto deste nutriente.

\section{AGRADECIMENTOS}

Ao Conselho Nacional de Desenvolvimento Científico e Tecnológico (CNPq) e a EMBRAPA pelo apoio em infraestrutura e logística na execução deste trabalho.

\section{REFERÊNCIAS}

ALCOBENDAS R et al. 2013. Effects of irrigation and fruit position on size, colour, firmness and sugar contents of fruits in a mid-late maturing peach cultivar. Scientia Horticulturae 164: 340-347.

ALVARES CA et al. 2013. Köppen's climate classification map for Brazil. Meteorologische Zeitschrift 22: 711-728.

BARRETO CF et al. 2017. Agronomic performance of the Maciel peach with different rootstocks. Semina: Ciências Agrárias 38: 1217-1228.

BRAND-WILLIANS $\mathrm{W}$ et al. 1995. Use of a free radical method to evaluate antioxidant activity. LebensmittelWissenschaft and Technologie 28: 25-30.

BRUNETTO $G$ et al. 2015. The pear tree response to phosphorus and potassium fertilization. Revista Brasileira de Fruticultura 37: 507- 516.

CHATZITHEODOROU IT et al. 2004. Effect of nitrogen, phosphorus, potassium fertilization and manure on fruit yield and fruit quality of the peach cultivars 'Spring Time' and 'Red Haven'. Agronomy Research 2: 135-143.

CQFS-RS/SC. 2016. Comissão de Química e Fertilidade do Solo. Manual de adubação e calagem para os estados do Rio Grande do Sul e Santa Catarina. 11.ed. Porto Alegre: SBCS. 376p.

CUQUEL FL et al. 2011. Nitrogen and potassium fertilization affecting the plum postharvest quality. Revista Brasileira de Fruticultura 33: 328-336.

DELGADO $\mathrm{R}$ et al. 2004. Changes in the phenolic composition of grape berries during ripening in relation vineyard nitrogen and potassium fertilization. Journal of the Science of Food and Agriculture 84: 623-630.

FANASCA S et al. 2006. Changes in antioxidant content of tomato fruits in response to cultivar and nutrient solution composition. Journal of Agricultural and Food Chemistry 54: 4319-4325.

FERREIRA DF. 2014. Sisvar: a Guide for its Bootstrap procedures in multiple comparisons. Ciência e Agrotecnologia 38 : 109-112.

FERREIRA LV et al. 2016. Qualidade de pêssegos submetidos à adubação nitrogenada. Revista Iberoamericana de Tecnología Postcosecha 17: 231-240.

FERREIRA LV et al. 2018. Nitrogen fertilization in consecutive cycles and its impact on high-density peach crops. Pesquisa Agropecuária Brasileira 53: 172-181.

GAZOLLA-NETO A et al. 2007. Qualidade do pêssego, cv. Maciel em função da adição de base mais foliar. Scientia Agraria 8: 233-237.

GANESHAMURTHY AN et al. 2011. Potassium nutrition on yield and quality of fruit crops with special emphasis on banana and grapes. Karnataka Journal of Agricultural Sciences 24: 29-38.

GONÇALVES MA et al. 2014. Qualidade de fruto e produtividade de pessegueiros submetidos a diferentes épocas de poda. Ciência Rural 44: 1334-1340.

JAWANDHA SK et al. 2017. Effect of potassium nitrate on fruit yield, quality and leaf nutrients content of plum. Vegetos An International Journal of Plant Research 30: 325-328.

JIFON JL \& LESTER GE. 2009. Foliar potassium fertilization improves fruit quality of field-grown muskmelon on calcareous soils in south Texas. Journal of the Science of Food and Agriculture 89: 2452-2460.

LESTER GE et al. 2010. Impact of potassium nutrition on postharvest fruit quality: Melon (Cucumis melo L.) case study. Plant Soil 335: 117-131.

MAYER NA et al. 2008. Qualidade pós-colheita de pêssegos de cultivares e seleções produzidos na microrregião de Jaboticabal-SP. Revista Brasileira de Fruticultura 30: 616-621.

MAYER NA et al. 2019. Pêssego, nectarina e ameixa: o produtor pergunta, a Embrapa responde. Brasília: Embrapa. $296 p$.

NAVA G et al. 2008. Nitrogen and potassium fertilization affect apple fruit quality in southern Brazil. Communications Soil and Plant Analysis 39: 96-107.

ROMBOLÀ AD et al. 2012. Nutrição e manejo do solo em fruteiras de caroço em regiões de clima temperado. Semina: Ciências Agrárias 33: 639-654.

SANTOS CM dos et al. 2013. Atividade antioxidante de frutos de quatro cultivares de pessegueiro. Revista Brasileira de Fruticultura 35: 339-344.

SOLHJOO S et al. 2017. Calcium and Potassium Foliar Sprays Affect Fruit Skin Color, Quality Attributes, and Mineral Nutrient Concentrations of 'Red Delicious' Apples. International Journal of Fruit Science 17: 358-373. 
SWAIN T \& HILLIS WE. 1959. The phenolic constituents of Prunus domestica. The quantitative analysis of phenolic constituents. Journal of the Science of Food and Agriculture 10: 63-68.

TAIZ L et al. 2017. Fisiologia e desenvolvimento vegetal. 6.ed. Porto Alegre: Artmed. 888p.

TALCOTT TS \& HOWARD RL. 1999. Phenolic autoxidation is responsible for color degradation in processed carrot puree. Journal of Agriculture and Food Chemistry 47: 2109-2115.

TREVISAN R et al. 2006. Uso de poda verde, plásticos refletivos, antitranspirante e potássio na produção de pêssegos. Pesquisa Agropecuária Brasileira 41: 1485-1490.

TREVISAN R et al. 2010. Perfil e preferências do consumidor de pêssego (Prunus persica) em diferentes regiões produtoras no Rio Grande do Sul. Revista Brasileira de Fruticultura 32: 90-100. 